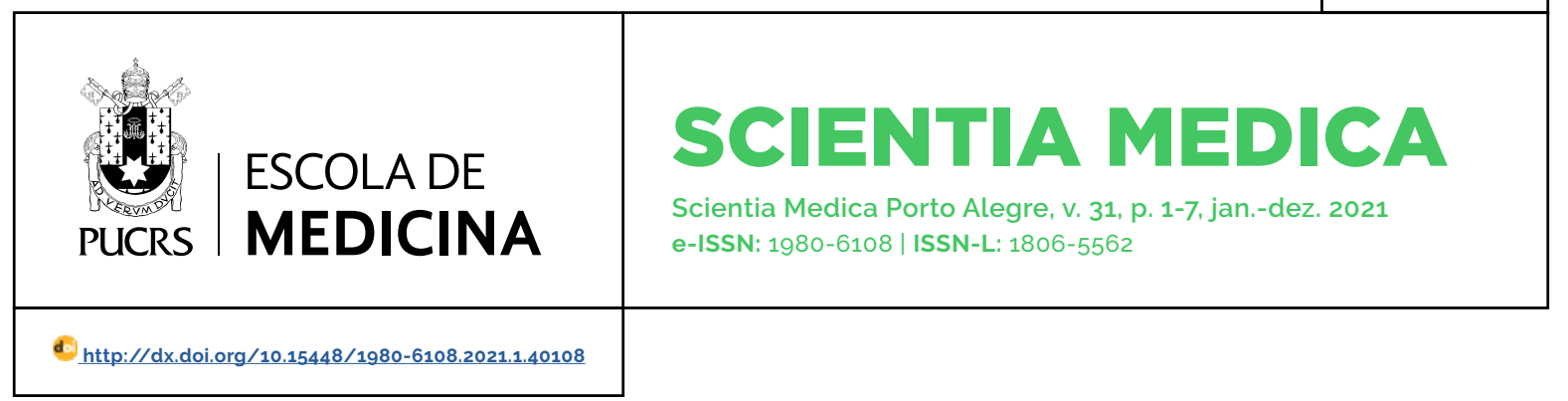

ARTIGO ORIGINAL

\title{
Perfil epidemiológico dos casos de toxoplasmose gestacional e congênita decorrentes do surto populacional
}

\author{
Epidemiological profile of gestational and congenital toxoplasmosis cases arising out \\ of the population outbreak
}

\author{
Natiele Camponogara Righi ${ }^{1}$ \\ orcid.org/0000-0001-7621-7204 \\ natirighi@gmail.com \\ Leticia Hermes ${ }^{2}$ \\ orcid.org/0000-0003-0331-6345 \\ letihermes1@gmail.com
}

Júlia Danezi Piccini orcid.org/0000-0001-8146-0591

juliadpiccini@gmail.com

\section{Jerônimo Costa Branco ${ }^{3}$}

orcid.org/0000-0001-6750-2740

jeronimobranco@hotmail.com

Jovito Adiel Skupien ${ }^{3}$

orcid.org/0000-0003-0892-3048

skupien.ja@gmail.com

\section{Angela Regina Maciel}

Weinmann ${ }^{1}$

orcid.org/0000-0003-2381-9396

noca@rocketmail.com

\section{Maria Clara da Silva \\ Valadão ${ }^{1}$ \\ orcid.org/0000-0002-6252-3152 mclaravalada02512@gmail.com}

Natielen Jacques Schuch ${ }^{3}$ orcid.org/0000-0001-7963-2104 natielen@yahoo.com.br

Recebido em: 6 fev. 2021. Aprovado em: 4 ago. 2021. Publicado em: 29 set. 2021.

\section{(c) (1)}

Artigo está licenciado sob forma de uma licença Creative Commons Atribuição 4.0 Internacional.

\section{Resumo:}

Objetivo: traçar o perfil epidemiológico dos casos de toxoplasmose gestacional e congênita notificados na cidade de Santa Maria - RS, decorrentes do surto ocorrido no ano de 2018 e investigar a associação entre as variáveis maternas e o diagnóstico do bebê.

Método: foram avaliados retrospectivamente os casos notificados entre o periodo de março de 2018 a março de 2019. O diagnóstico do bebê e variáveis maternas foram analisados através de estatística descritiva e teste qui-quadrado.

Resultados: Durante o periodo do estudo, foram notificados 206 casos de toxoplasmose gestacional, dos quais 74 foram confirmados como infecção recente. A transmissão vertical foi confirmada em $27 \%$ dos casos. Houve maior freqüência de transmissão em gestantes que tiveram o diagnóstico no terceiro trimestre de gestação $(p=0,001)$ e que não realizaram tratamento durante a gestação $(p<0,001)$.

Conclusão: A ocorrência da toxoplasmose congênita está associada ao diagnóstico tardio, no último trimestre da gestação, e consequente falta do tratamento adequado. Ainda, houve uma alta prevalência de crianças infectadas por transmissão vertical decorrentes do surto, o que reforça a importância de um acompanhamento pré-natal e a atenção à necessidade da realização do tratamento e cuidado adequados no decorrer do desenvolvimento das crianças infectadas.

Palavras-chave: toxoplasmose congênita, gestação, epidemiologia.

\section{Abstract}

Objective: To trace the epidemiological profile of cases of gestational and congenital toxoplasmosis reported in the city of Santa Maria - RS, resulting from the outbreak that occurred in 2018 and to investigate the association between maternal variables and the baby's diagnosis.

Method: Cases reported between March 2018 and March 2019 were retrospectively evaluated. The baby's diagnosis and maternal variables were analyzed using descriptive statistics and chi-square test.

Results: During the study period, 206 cases of gestational toxoplasmosis were reported, of which 74 were confirmed as a recent infection. Vertical transmission was confirmed in $27 \%$ of cases. There was a higher frequency of transmission in pregnant women who were diagnosed in the third trimester of pregnancy $(p=$ $0.001)$ and who did not undergo treatment during pregnancy $(p<0.001)$.

Conclusion: The occurrence of congenital toxoplasmosis is associated with late diagnosis, in the last trimester of pregnancy, and consequent lack of adequate treatment. In addition, there was a high prevalence of children infected by vertical transmission due to the outbreak, which reinforces the importance of prenatal care and attention to the need for adequate treatment and care during the development of infected children.

Keywords: toxoplasmosis congenital, pregnancy, epidemiology. 


\section{Introdução}

A toxoplasmose é definida como o resultado da infecção pelo parasita Toxoplasma gondii, que tem como seu principal hospedeiro o gato. Porém, durante seu ciclo de vida pode passar pelo ser humano, que vai atuar como um hospedeiro intermediário $(1,2)$. A infecção por esse parasita durante a gestação pode ser sintomática ou, como em 90\% dos casos, assintomática, e pode levar à transmissão transplacentária (3). A gravidade da transmissão vertical depende do trimestre da gravidez em que a mãe é infectada (3), sendo inversamente proporcional ao avanço da gestação (3). A sequela mais comum relacionada à toxoplasmose congênita é a perda de visão, podendo ocorrer também corior retinite, calcificação intracerebral, hidrocefalia, retardo mental e perda auditiva $(3,4)$.

O diagnóstico é feito através da detecção de anticorpos especificos para o Toxoplasma, a Imunoglobulina G (lgG) e a Imunoglobulina M (IgM) (2, 4), este último confirmando uma exposição aguda (2). Quando confirmada ou suspeita a infecção aguda na gravidez, a mulher deve iniciar o tratamento o mais breve possivel, diminuindo, assim, os riscos de infecção fetal (4). O tratamento tem por objetivo interromper a replicação do parasita e, assim, evitar novos danos aos órgãos envolvidos (2). Ainda que o tratamento seja aplicado durante a gestação, há possibilidade de ocorrer a transmissão vertical (5).

É estimado que, tanto em paises desenvolvidos como em desenvolvimento, um terço da população mundial tenha sido infectada pelo parasita (5). No Nordeste do Brasil, um estudo realizado em dois centros de referência para pré-natal de alto risco apresentou alta $(77,9 \%$ ) sororeatividade para toxoplasmose entre as 561 pacientes atendidas no período de julho de 2011 a dezembro de 2012 (6). A prevalência de toxoplasmose em gestantes atendidas em uma maternidade da cidade de Porto Alegre, no Rio Grande do Sul, nos meses de julho a outubro e dezembro do ano de 2000 , que envolveu 1.261 gestantes, foi de 59,8\% (7). Já em unidades de saúde de municipios do Noroeste do Rio Grande do Sul, em uma população de 2.126 gestantes, foi de $74.5 \%$, no periodo de julho de 1997 a dezembro de 1998 (8). A partir de um estudo realizado entre os anos de $1995 \mathrm{e}$ 2009, Neto, Amorim e Lago (2010) observaram que a prevalência de toxoplasmose congênita no Brasil é de 1/1.613 (9).

Em várias partes do mundo, foram descritos surtos de toxoplasmose (10). No ano de 2018, a cidade de Santa Maria, no Rio Grande do Sul apresentou uma elevação aguda da incidência de casos de toxoplasmose na população (11s). Por esse motivo, o objetivo do presente estudo foi traçar o perfil epidemiológico dos casos de toxoplasmose aguda gestacional e congênita notificados na cidade de Santa Maria, RS, no periodo de um ano e investigar a associação entre as variáveis maternas e o diagnóstico do bebê.

\section{Métodos}

Trata-se de um estudo transversal retrospectivo, aprovado pelo Comitê de Ética em Pesquisa da Universidade Federal de Santa Maria sob parecer n. ${ }^{\circ}$ 3.254.507, realizado no periodo de março de 2018 a março de 2019. O estudo está reportado de acordo com o Strengthening the Reporting of Observational studies in Epidemiology (Strobe Statement).

A amostra foi constituida por conveniência, pelas fichas de notificação compulsória dos casos de toxoplasmose gestacional e congênita notificados ao Núcleo de Vigilância Epidemiológica do Hospital Universitário de Santa Maria, na cidade de Santa Maria, Rio Grande do Sul, e prontuários eletrônicos.

Para ser considerada toxoplasmose gestacional, a infecção deveria ser recente, ou seja, apresentar anticorpos IgM especificos para Toxoplasma gondii e avidez de lgG baixa (<30\%)(12), dessa forma, todos prontuários que apresentavam esse diagnóstico foram incluídos no estudo. Aqueles onde não foi possivel fazer a identificação pela falta de informação e/ou erro de preenchimento foram excluídos do estudo.

As variáveis foram coletadas diretamente da ficha e do prontuário eletrônico dos pacientes. As variáveis maternas coletadas foram: faixa etária, estado civil, bairro onde residiam, tipo 
de parto, trimestre em que foi diagnosticada a toxoplasmose, sintomas (febre, mialgia, cefaleia, linfadenomegalia) e realização de tratamento. Para a categorização dos bairros onde as gestantes residiam, foi utilizada a divisão por regiões administrativas da cidade de Santa Maria.

Em relação ao desfecho, foram coletados dados relacionados ao diagnóstico do bebê: recém-nascido ( $R N$ ) vivo toxoplasmose congênita, RN vivo exposto, investigação, perda de seguimento ou aborto. A confidencialidade e o anonimato dos dados coletados foram assegurados.

A análise dos dados foi realizada através do pacote estatístico IBM SPSS Statistics 22. Foi realizada análise descritiva. Os dados estão apresentados em média \pm desvio padrão ou em número e percentuais, e o teste qui-quadrado foi conduzido para determinar a relação das variáveis maternas com o diagnóstico do bebê com um nivel de significância de 5\%.

\section{Resultados}

No periodo do estudo foram notificados 206 casos de toxoplasmose gestacional, sendo todos analisados no estudo. Entretanto, desses, apenas 74 (35.9\%) foram confirmados como infecção recente/toxoplasmose adquirida na gestação. A Tabela 1 apresenta as variáveis maternas investigadas nas fichas de notificação compulsória e prontuários dos casos de toxoplasmose gestacional e congênita do Hospital Universitário de Santa Maria, em Santa Maria, RS. A idade média das gestantes foi de $28 \pm 6,9$ anos. Com relação ao bairro em que as gestantes residiam, a região Central concentrava 16,2\% das gestantes incluídas, 9.5\% estavam nas regiões Sul e Norte-Nordeste, 59,5\% no Oeste-Centro Oeste e 5,4\% no Leste-Centro Leste da cidade de Santa Maria.
TABELA 1 - Variáveis maternas investigadas nas fichas de notificação compulsória e prontuários dos casos de toxoplasmose gestacional e congênita do Hospital Universitário de Santa Maria, Santa Maria-RS

$n(\%)$

$\begin{array}{ll}\text { Faixa etária } & \\ 16-19 \text { anos } & 9(12,2) \\ 20-34 \text { anos } & 51(68,9) \\ 35 \text { anos ou mais } & 14(18,9) \\ \text { Estado Civil } & \\ \text { Solteira } & 50(67,6) \\ \text { Casada ou em união estável } & 24(32,4)\end{array}$

Parto

Vaginal

$44(59.5)$

Cesariana

$18(24,3)$

Aborto

$4(5,4)$

Desconhecido

$8(10,8)$

Trimestre de diagnóstico

$24(32,4)$

$2^{\circ}$

$19(25,7)$

$3^{\circ}$

$31(41,9)$

Sintomas de toxoplasmose

Sim

$23(31,1)$

Não

$33(44,6)$

Sem informação

$18(24,3)$

Tratamento

Sim

$58(78,4)$

Diagnóstico do recém-nascido

Com Toxoplasmose Congênita

20 (27)

Exposto à Toxoplasmose Congênita

$23(31,1)$

Em investigação

$11(14,9)$

Perda de seguimento

$13(17,6)$

Aborto

$7(9,5)$ 
Da amostra, 23 gestantes apresentaram sintomas. Dentre elas, 21,6\% tiveram febre, 23\% mialgia, 29,3 cefaléia e 36,5\% apresentaram linfadenomegalia. Com relação ao tratamento realizado, 21,6\% utilizaram Espiramicina, 32,4\% utilizaram esquema SPAF (Sulfadiazina, Pirimetamina e Ácido Folínico) e 21,6\% iniciaram com Espiramicina e posteriormente passaram para esquema SPAF. A transmissão vertical foi confirmada em $27 \%$ dos casos.

Houve associação entre o trimestre de diagnóstico e a toxoplasmose congênita $(p=0,001)$, sendo mais frequente a transmissão em gestantes que tiveram o diagnóstico no terceiro trimestre de gestação. A maior frequência de transmissão vertical e infecção também está associada com a não realização de tratamento durante a gestação $(p<0,001)$ e, dentre as formas de tratamento utilizadas (Espiramicina, SPAF e Espiramicina e após SPAF), ambas foram eficazes. As demais variáveis analisadas não apresentaram associação com o diagnóstico do bebê ( $p>0,05)$.

\section{Discussão}

A cidade de Santa Maria, RS passou por um surto de casos de toxoplasmose no ano de 2018 (11). Dentre os casos notificados, observou-se a ocorrência também de toxoplasmose gestacional, que pode ser transmitida para a criança (3). O presente estudo demonstrou que a ocorrência de toxoplasmose congênita está associada ao trimestre de diagnóstico e à realização de tratamento da toxoplasmose gestacional.

A idade média materna do presente estudo (28 anos) foi maior do que a encontrada em estudo prévio realizado em gestantes submetidas à triagem pré-natal em todo o estado de Mato Grosso do Sul (Brasil), que foi de 23 anos (13). Assim como nos resultados apresentados, Figueiró-Filho et al. (13) também observaram que não há relação entre a faixa etária materna e a infecção aguda pelo Toxoplasma gondii. Porém, estudos anteriores demonstraram que existe uma tendência para o aumento de soropositividade com o aumento da idade materna $(7,14)$.

Estudo prévio realizado na Noruega determinou que hábitos alimentares, fatores culturais, regiões geográficas e características climáticas são fatores de risco específicos para infecção pelo Toxoplasma gondii durante a gestação (15). Mais de 50\% das gestantes identificadas com toxoplasmose gestacional derivadas do surto, no período do estudo, residiam na região administrativa Oeste-Centro Oeste de Santa Maria, RS. Porém, nenhuma região onde as gestantes residiam apresentou associação com o diagnóstico do bebê.

A forma com que o Toxoplasma gondii se apresenta na infecção aguda são os taquizoítos, que se multiplicam rapidamente e atravessam a placenta através da circulação materna (165, entrando na circulação fetal (5). A taxa de transmissão vertical da toxoplasmose é diretamente proporcional à idade gestacional em que a mãe foi infectada $(17,18)$, o que pode ser explicado por alterações fenotípicas que ocorrem no desenvolvimento da placenta, fazendo com que suas células sejam mais permissivas à infecção quanto mais tardia for a gestação (19). Neste sentido, no presente estudo, a maior ocorrência de toxoplasmose congênita foi associada ao diagnóstico no terceiro trimestre de gestação. Reforçando esse fato, em uma coorte realizada na Itália entre os anos de 2001 a 2012, houve maior incidência de toxoplasmose congênita nas crianças em que a mãe foi infectada no terceiro trimestre de gestação (20).

O tratamento da gestante também está associado ao diagnóstico do bebê, sendo a transmissão vertical mais frequente nos casos em que a gestante não realizou o tratamento. Segundo a Federação Brasileira das Associações de Ginecologia e Obstetrícia, a Espiramicina é o antibiótico de escolha inicial, que vai atuar sobre a infecção placentária e deve ser mantido quando o exame da reação em cadeia da polimerase (PCR) der resultado negativo. Já em caso de PCR positiva, que indica infecção fetal, deve-se substituir a Espiramicina pelo esquema SPAF, que atua eliminando os agentes que atravessaram a barreira placentária (17, 21). O tipo de tratamento utilizado pela amostra do presente estudo não foi um fator associado à transmissão vertical, sendo menor a frequência dessa independentemente do tratamento realizado. Da mesma maneira, 
recente ensaio clínico comparou a utilização da Espiramicina e do esquema SPAF na redução da transmissão placentária da toxoplasmose, não observando diferença entre os tratamentos (22).

Como limitação do estudo, é destacado o fato de não ter sido realizada entrevista com as gestantes. Como os dados foram extraidos apenas através de fichas pré-existentes e prontuário eletrônico, muitas informações não foram encontradas, principalmente no que se refere à sintomatologia da toxoplasmose gestacional. Porém, as associações encontradas no presente estudo ratificam a importância do acompanhamento pré-natal das gestantes e das medidas preventivas primárias contra a toxoplasmose, como adoção de hábitos higiênicos e alimentares adequados, principalmente para as gestantes soronegativas que iniciam o pré-natal. Também é importante sensibilizar e capacitar os profissionais de saúde para que sejam passadas informações corretas para as gestantes, tendo em vista que, muitas vezes, o diagnóstico da toxoplasmose acontece no terceiro trimestre e não há tempo hábil para o tratamento da gestante, ocasionando a transmissão vertical do parasito. Ainda, é fundamental que a criança seja acompanhada, principalmente durante o primeiro ano de vida, mesmo que não apresente toxoplasmose congênita confirmada (21). Esse estudo poderá auxiliar na organização de estratégias de acompanhamento, controle e prevenção de toxoplasmose gestacional e congênita.

\section{Conclusão}

A ocorrência da toxoplasmose congênita está associada ao diagnóstico tardio, no último trimestre da gestação, e consequente falta do tratamento adequado. Ainda, houve uma alta prevalência de crianças infectadas por transmissão vertical decorrentes do surto, o que reforça a importância do acompanhamento pré-natal e da prevenção primária com as gestantes soronegativas, como também da realização do tratamento e do cuidado adequados no decorrer do desenvolvimento das crianças infectadas. Evidenciou-se por esse estudo a necessidade de treinamento dos profissionais que preenchem as fichas de notificação, uma vez que as informações completas são necessárias para o processo de organização de estratégias de acompanhamento, controle e prevenção da toxoplasmose gestacional e congênita.

\section{Notas}

\section{Apoio financeiro}

Este estudo não recebeu apoio financeiro de fontes externas.

\section{Declaração de conflito de interesses}

Os autores declaram não haver conflitos de interesses relevantes ao conteúdo deste estudo.

\section{Contribuições dos autores}

Todos os autores fizeram contribuições substanciais para concepção, ou delineamento, ou aquisição, ou análise ou interpretação de dados; e redação do trabalho ou revisão crítica; e aprovação final da versão para publicação.

\section{Disponibilidade dos dados e responsabilidade pelos resultados}

Todos os autores declaram ter tido total acesso aos dados obtidos e assumem completa responsabilidade pela integridade destes resultados.

\section{Referências}

1. Guy EC. Toxoplasmosis. Medicine (Baltimore) 2014;42(1):31-3.

2. Kaye A. Toxoplasmosis: Diagnosis, Treatment, and Prevention in Congenitally Exposed Infants. J Pediatr Health Care. 2011;25(6):355-64. https://doi.org/10.1016/j. pedhc.2010.04.008

3. Kravetz JD, Federman DG. Toxoplasmosis in pregnancy. Am J Med. 2005;118:212-6. https://doi. org/10.1016/j.amjmed.2004.08.023

4. Singh S. Congenital toxoplasmosis: Clinical features, outcomes, treatment, and prevention. Trop Parasitol. 2016;6(2):113-22. https://doi.org/10.4103/22295070.190813

5. Montoya JG, Liesenfeld O. Toxoplasmosis. Lancet. 2004:363:1965-76. https://doi.org/10.1016/s01406736(04)16412-X 
6. Câmara JT, da Silva MG, de Castro AM. Prevalência de toxoplasmose em gestantes atendidas em dois centros de referência em uma cidade do Nordeste, Brasil. Rev Bras Ginecol Obstet. 2015:37(2):64-70. https:// doi.org/10.1590/SO100-720320150005115

7. Varella IS, Wagner MB, Darela AC, Nunes LM, Müller RW. Prevalência de soropositividade para toxoplasmose em gestantes. J Pediatr (Rio J). 2003;79(1):69-74. https:// doi.org/10.1590/S0021-75572003000100012

8. Spalding SM, Amendoeira MRR, Ribeiro LC, Silveira C, Garcia AP, Camilo-Coura L. Estudo prospectivo de gestantes e seus bebês com risco de transmissão de toxoplasmose congênita em município do Rio Grande do Sul. Rev Soc Bras Med Trop. 2003:36(4):483-91. https://doi.org/10.1590/S0037-86822003000400009

9. Neto EC, Amorim F, Lago EG. Estimation of the regional distribution of congenital toxoplasmosis in Brazil from the results of neonatal screening [Internet]. Sci Med (Porto Alegre). 2010 [citado 2021 Jul 10];20(1):6470. Disponível em: https://revistaseletronicas.pucrs.br/ ojs/index.php/scientiamedica/article/view/5983/4877

10. Maldonado YA, Read JS. Diagnosis, Treatment, and Prevention of Congenital Toxoplasmosis in the United States. Pediatrics. 2017;139(2):e20163860. https://doi. org/10.1542/peds.2016-3860

11. Rio Grande do Sul. Secretaria de Saúde. Alerta Epidemiológico. Toxoplasmose [Internet]. 2018 [citado 2021 Jul 10]. Disponivel em: https://saude.rs.gov.br/ upload/arquivos/carga20180752/25125245-09144313o.9-05-18-alerta-toxoplamose.pdf

12. Jenum PA, Stray-Pedersen B, Gunderser A-G. Improved Diagnosis of Primary Toxoplasma gondii Infection in Early Pregnancy by Determination of Antitoxoplasma Immunoglobulin G Avidity. J Clin Microbiol. 1997:35(8):1972-7. https://doi.org/10.1128/ jcm.35.8.1972-1977.1997

13. Figueiró-Filho EA, Lopes AHA, Senefonte FR de $A$, de Souza Júnior VG, Botelho CA, Figueiredo MS, et al. Toxoplasmose aguda: estudo da freqüência, taxa de transmissão vertical e relação entre os testes diagnósticos materno-fetais em gestantes em estado da Região Centro-Oeste do Brasil. Rev Bras Ginecol Obstet. 2005;27(8):442-9. https://doi.org/10.1590/ S0100-72032005000800002

14. Avelar JB, da Silva MG, Rezende HHA, Storchilo $\mathrm{HR}$, do Amaral WN, Xavier IR, et al. Epidemiological factors associated with Toxoplasma gondii infection in postpartum women treated in the public healthcare system of Goiânia, State of Goiás, Brazil. Rev Soc Bras Med Trop. 2018:51(1):57-62. https://doi. org/10.1590/0037-8682-0112-2017

15. Kapperud G, Jenum PA, Stray-pedersen B, Melby KK, Eskild A, Eng J. Risk Factors for Toxoplasma gondii Infection in Pregnancy: Results of a Prospective CaseControl Study in Norway. Am J Epidemiol. 1996;144(4):40512. https://doi.org/10.1093/oxfordjournals.aje.a008942
16. Walcher DL, Comparsi B, Pedroso D. Toxoplasmose gestacional: uma revisão. RBAC. 2017:49(4):323-7. https://dx.doi.org/10.21877/2448-3877.201600273.

17. Federação Brasileira das Associações de Ginecologia e Obstetricia (FEBRASGO) Manual de Gestação de Alto Risco [Internet]. 2011 [citado 2021 Jul 10]; 220 p. disponivel em: http://Www.as.saude.ms.gov.br/wp-content/ uploads $/ 2019 / 08 /$ manual-de-gesta\%c3\%87\%c3\%830de-alto-risco-2011.pdf

18. Dunn D, Wallon M, Peyron F, Petersen E, Peckham C. Gilbert R. Mother-to-child transmission of toxoplasmosis: risk estimates for clinical counselling. Lancet. 1999;353(9167):1829-33. https://doi.org/10.1016/ S0140-6736(98)08220-8

19. Gomes ADO, Silva DAO, Silva NM, Barbosa BDF Franco PS, Angeloni MB, et al. Effect of Macrophage Migration Inhibitory Factor (MIF) in Human Placental Explants Infected with Toxoplasma gondii Depends on Gestational Age. Am J Pathol. 2011;178(6):2792-801. https://doi.org/10.1016/j.ajpath.2011.02.005

20. Donadono V, Saccone G, Giuseppe MM, Berghella V, Migliorini S, Esposito G, et al. Incidence of toxoplasmosis in pregnancy in Campania: A population-based study on screening, treatment, and outcome. Eur J Obstet Gynecol Reprod Biol. 2019;240:316-21. https://doi. org/10.1016/j.ejogrb.2019.07.033

21. Paraná. Secretaria de Estado da Saúde. Caderno atenção ao pré-natal - Toxoplasmose [Internet]. Curitiba: Secretaria de Estado da Saúde [citado 2021 Jul 10]. Disponivel em: http://www.sjp.pr.gov.br/wpcontent/uploads/2018/04/ANEXO-VII-toxoplasmosecong\%C3\%AAnita.pdf

22. Mandelbrot L, Winer N, Mesnard L, Berrebi A, Bory J, Ville Y, et al. Prenatal therapy with pyrimethamine + sulfadiazine vs spiramycin to reduce placental transmission of toxoplasmosis: a multicenter, randomized trial. Am J Obstet Gynecol. 2018;219:386. e1-9. https://doi.org/10.1016/j.ajog.2018.05.031

\section{Natiele Camponogara Righi}

Mestre em Reabilitação Funcional pela Universidade Federal de Santa Maria (UFSM), em Santa Maria, RS, Brasil.

\section{Letícia Hermes}

Mestre em Ciências da Saúde pela Universidade Federal de Santa Maria (UFSM), em Santa Maria, RS, Brasil.

\section{Júlia Danezi Piccini}

Pediatra pelo Hospital Universitário de Santa Maria (HUSM) e Universidade Federal de Santa Maria (UFSM), em Santa Maria, RS, Brasil. 


\section{Jerônimo Costa Branco}

Doutor em Saúde e Comportamento pela Universidade Católica de Pelotas. Docente na Universidade Franciscana (UFN), em Santa Maria, RS, Brasil.

\section{Jovito Adiel Skupien}

Doutor em Odontologia pela Universidade Federal de Pelotas (UFPel). Docente na Universidade Franciscana (UFN), em Santa Maria, RS, Brasil.

\section{Angela Regina Maciel Weinmann}

Doutora pela Faculdade de Medicina de Ribeirão Preto, Universidade de São Paulo (FMRP-USP). Docente na Universidade Franciscana (UFN) e na Universidade Federal de Santa Maria (UFSM), em Santa Maria, RS, Brasil.

\section{Maria Clara da Silva Valadão}

Doutora em Medicina/Pediatria pela Pontificia Universidade Católica do Rio Grande do Sul (PUCRS), em Porto Alegre, RS, Brasil. Docente na Universidade Franciscana (UFN) em Santa Maria, RS, Brasil.

\section{Natielen Jacques Schuch}

Doutora em Nutrição em Saúde Pública pela Faculdade de Saúde Pública da Universidade de São Paulo. Docente na Universidade Franciscana (UFN) em Santa Maria, RS, Brasil.

\section{Endereço para correspondência}

Natielen Jacques Schuch

Universidade Franciscana

Rua dos Andradas, 1614, prédio 16, sala 209

Centro, 97010-032

Santa Maria, RS, Brasil

Os textos deste artigo foram revisados pela Poá Comunicação e submetidos para validação do(s) autor(es) antes da publicação. 